\title{
Questions de communication
}

\section{Stefan GOLTZBERG, Chaïm Perelman. L'argumentation}

juridique

Paris, Michalon, coll. Le Bien commun, 2013, 119 pages

Julien Mumpwena Nsulung

\section{OpenEdition}

\section{Journals}

Édition électronique

URL : http://journals.openedition.org/questionsdecommunication/9957

DOI : 10.4000/questionsdecommunication.9957

ISSN : 2259-8901

Éditeur

Presses universitaires de Lorraine

Édition imprimée

Date de publication : 1 septembre 2015

Pagination : 412-414

ISBN : 9782814302600

ISSN : 1633-5961

Référence électronique

Julien Mumpwena Nsulung, "Stefan goltzberg, Chaïm Perelman. L'argumentation juridique », Questions de communication [En ligne], 27 | 2015, mis en ligne le 01 septembre 2015, consulté le 10 décembre 2020. URL : http://journals.openedition.org/questionsdecommunication/9957 ; DOI : https://doi.org/ 10.4000/questionsdecommunication.9957 

Stefan GoltzBerg, Chaïm Perelman. L'argumentation
juridique.

Paris, Michalon, coll. Le Bien commun, 2013, I 19 pages

Pour qui s'intéresse à l'argumentation juridique, il devient aujourd'hui difficile de faire l'économie des intuitions de Chaïm Perelman. Pour marquer le $100^{e}$ anniversaire de la naissance de cette grande figure de la théorie du droit du Xx siècle, Stefan Goltzberg offre l'occasion d'en saisir la quintessence.

Pour l'auteur, l'argumentation juridique doit faire l'objet d'un enseignement à part entière. II regrette que « dans la plupart des lieux où l'on enseigne le droit,l'argumentation juridique ne fait pas l'objet d'une théorisation explicite », mais soit transmise « par mimétisme » (p.8). Ce livre veut combler, à sa manière, cette lacune. D'où sa dimension pédagogique et critique. L'auteur est convaincu qu'une compréhension du mécanisme ou des procédés mis en œuvre par les hommes de droit « peut aider à combler le fossé qui tend à séparer les professionnels de l'argumentation juridique et les justiciables dont le sort bien souvent dépendra du type d'arguments retenu par le juge » (pp. 9-10).

Stefan Goltzberg divise sa réflexion en trois chapitres. Le premier (« Au-delà du positivisme », pp. 13-33) présente le rapport conflictuel qu'entretient Chaïm Perelman avec le positivisme ; le deuxième (« La Nouvelle Rhétorique », pp. 35-49) expose l'apport de Chaïm Perelman à la rhétorique et à l'argumentation et le troisième («L'Argumentation juridique », pp. 51-93), est l'objet même du livre. Qu'en est-ll ?

Dans le premier chapitre, le chercheur rappelle que la philosophie de Chaïm Perelman, en particulier sa théorie de l'argumentation, tout en étant bâtie contre le positivisme, propose un « au-delà du positivisme ». En effet, le positivisme regroupe l'ensemble des écoles qui, non seulement séparent le fait et la valeur (p. I3), mais rejette aussi la cause finale, en se voulant être axiologiquement neutre. À en croire Stefan Goltzberg, « cette neutralité axiologique, bien qu'elle ait défini la Modernité, est remise en cause par la Postmodernité » (pp. 13-14). Toutefois, ce qui l'intéresse, c'est la compréhension perelmanienne du positivisme juridique : le rapport entre le droit et la loi. Ce rapport est tel que, pour le positiviste, le droit se limite à la loi et que cette dernière est exhaustive. Elle prévoit tout, en attribuant au juge une fonction précise : l'application mécanique de la loi au cas d'espèce (p. I5). II est donc soumis à la loi. Ainsi une des conséquences est-elle la subordination du judiciaire au législatif. Le juge ne joue aucun rôle dans l'interprétation de la loi. II n'est pas source du droit. Par conséquent, il n'y a pas de place pour les 《 valeurs », lesquelles sont considérées comme arbitraires, « le lit de l'irrationnel préscientifique » (p. 16), et donc déraisonnables. Stefan Goltzberg passe en revue ces notions, « valeur 》, « arbitraire 》, « raisonnable »/« déraisonnable » et le rôle qu'elles jouent dans la pensée juridique de Chaïm Perelman.

Tout en partageant avec le positivisme le rejet de l'arbitraire - concept dont le contenu est à spécifier -, Chaïm Perelman ne pense pas maintenir les valeurs hors du champ de l'argumentation. Pour ce dernier, le droit ne peut avoir comme seule source la loi, même si l'on peut assister à un conflit ou à des désaccords entre ses différentes sources. Mais pour Chaïm Perelman, ces désaccords ne sont pas signes d'irrationalité, ils sont légitimes. C'est pourquoi il « célèbre le désaccord comme un fait noble et légitime, qui ouvre le débat et le rend possible. La démocratie suppose la possibilité du désaccord $\gg($ p. $\mid 8)$.

On comprend alors que même la sécurité juridique, en ce sens, n'est plus un dogme, mais une valeur. Dès lors, le système juridique ne peut se comporter comme un système axiomatique formalisé : fermé, complet, univoque et cohérent. Car, dans la mesure où le droit continue à s'exprimer en langue naturelle, on ne peut éviter ou écarter les ambiguités ou les notions floues. Ainsi Chaïm Perelman trouve-t-il « dans les défauts soi-disant rédhibitoires de l'argumentation juridique, les conditions de possibilité d'une métorique judiciaire qui fasse droit à la justice elle-même » (p. 20). C'est pourquoi, pour lui, «le système du droit est ouvert et doit le rester » (p. 2l).

Stefan Goltzberg relève en outre que le positivisme juridique s'est manifesté à travers deux écoles: d'une part, l'École de l'exégèse et, d'autre part, celle conduite par Hans Kelsen. Pour la première école, « très attachée à la séparation des pouvoirs et à la soumission du pouvoir judiciaire au pouvoir législatif » (p. 22), « la clarté s'oppose à l'interprétation 》 (p. 23). Or, c'est justement cette notion « d'un texte clair » qui pose problème pour Chaïm Perelman. Pour lui, « clair est un compliment que l'on fait à un texte dans l'exacte mesure où il n'est pas discuté » (p. 23) ; ce n'est donc pas une propriété objective du texte. Il en donne pour preuve l'existence des antinomies et des lacunes qui permettent aux juges de contribuer à l'élaboration de la règle de droit. Ces deux exceptions constituent justement la faille de cette école. Toutefois, selon Stefan Goltzberg, « l'existence d'une lacune n'est pas tant un fait constatable qu'une décision » (p. 27), ce que Chaïm Perelman ne signale pas assez.Ainsi, pour ce dernier, la 
loi étant l'une des sources du droit, «l'esprit de la loi doit être soumis à l'esprit du droit » (p. 29).

Quant au dialogue avec Hans Kelsen, représentant de la deuxième école du positivisme, le chercheur se limite à fournir un résumé de la position de Chaimm Perelman. On aurait souhaité une présentation, même brève, de celle d'Hans Kelsen à laquelle s'attaque Chaïm Perelman. Mais on comprend que c'est la lecture de Chaïm Perelman qui importe ici. En effet, pour lui, Hans « Kelsen construit sa philosophie du droità partir d'une hiérarchie des normes, souvent représentée sous la forme d'une pyramide » (p. 29), où « chaque norme serait déduite de la de la norme et la norme fondamentale, la Grundnorm est ellemême présupposée » (p. 29). Ce qui interdit l'étude des jugements de valeurs. Ces derniers sont exclus du champ du rationnel et du connaissable. Or, c'est justement la mise à l'honneur des valeurs qui prouve l'importance de la Nouvelle Rhétorique. II faut donc « réinstaller les valeurs dans le champ de l'argumentation juridique » (p. 33) afin de s'assurer du fonctionnement de la justice.

Stefan Goltzberg traite de la Nouvelle Rhétorique en exploitant la Logique juridique de Chaim Perelman, et le chapitre intitulé « La Nouvelle Rhétorique et les valeurs ». Lídée de base est que la Nouvelle Rhétorique est « intimement liée aux valeurs » (p. 36). Pour l'expliquer, on peut recourir à l'analogie perelmanienne entre la rhétorique et la langue naturelle ou technique: celle-ci n'est ni nécessaire, ni arbitraire. À en croire Stefan Goltzberg, Chaïm Perelman estime, à ce sujet, qu'il faut éviter deux écueils : la croyance que la langue reflète objectivement le monde d'une seule manière possible; et la réduction du choix des mots à un choix arbitraire (p. 36). Pour lui, une langue est sous-tendue par des valeurs qui la structurent, de sorte que son apprentissage signifie adhésion à celles-ci.

On comprend alors que Chaïm Perelman place les valeurs au cœur de sa Nouvelle Rhétorique. Comprise comme « techniques discursives visant à provoquer ou à accroitre l'adhésion des esprits aux thèses que l'on présente à leur assentiment » (p. 37), cette dernière exclut « les actions comme la violence et la caresse » (p. 37) : « La rhétorique trouve sa raison d'être dans le fait que les esprits ont besoin d'être convaincus » (p. 37). Pour y arriver, l'évidence seule en est incapable, surtout dans des domaines autres que ceux des sciences formalisées. Aussi, écrit Chaïm Perelman, cette rhétorique « ne concerne pas tant la vérité que l'adhésion » (cité p. 38). Stefan Goltzberg précise ici que « la définition [de la rhétorique] n'évoque pas un discours visant à nous convaincre de la vérité d'une thèse, mais une adhésion des esprits » (p. 38).
En outre, La Nouvelle Rhétorique englobe l'idée d'auditoire, lequel, tout en étant une construction, est « sensible aux arguments et non aux seules preuves de type mathématique » (p. 39). Cet auditoire peut alors prendre en considération le conflit des valeurs. Signalons ici que, pour Chaïm Perelman, la vérité est considérée comme une valeur, laquelle est « tantôt opposée aux valeurs et [...] tantôt constitue une valeur elle-même, certes prépondérante » (p. 40).

Le projet de la Nouvelle Rhétorique est alors un refus du formalisme, refus d'un discours more geometrico, lequel est binaire, contraignant, mécanique, indépendant du contexte, homogène et repose sur la centralité de la vérité (pp. 41-44). La rhétorique ou l'argumentation met en exergue les lieux qui « sont des raisons que l'on fait valoir pour convaincre son auditoire » (p. 45). Ces lieux sont aussi propres à un domaine, à un groupe. lls peuvent aussi être universels. Parmi eux, il y a les lieux communs qui relèvent du sens commun. Ces derniers peuvent être ambigus. Ce qui leur permet « de ne pas être invalidés par d'autres lieux communs » (p. 46). Ils demeurent valides d'une controverse à l'autre. D'où la nécessité, non seulement de prendre en compte le contexte de l'argumentation, mais aussi de connaitre les lieux partagés par l'auditoire afin de les rendre présents. Ces lieux de l'argumentation sont importants dans l'argumentation juridique, même s'ils ne sont partagés que par les hommes de droit. Ainsi, à en croire Stefan Goltzberg, les lieux de l'argumentation sont-ils « une pièce centrale dans la rhétorique » (p.48). On leur doit une attention particulière. Mais, qu'en est-il finalement de l'argumentation juridique perelmanienne? Tel est le nœud du livre.

L'objet du troisième chapitre est celui de la spécificité de l'argumentation juridique par rapport à la rhétorique en général, ce que Chaim Perelman entend par logique juridique et l'analyse de quelques types d'arguments.

En effet, entre l'argumentation philosophique et l'argumentation juridique, il y a un enrichissement mutuel. Alors que « les philosophes restent volontiers dans l'abstraction et le général » (p. 52), à peser les pour et les contre sans peut-être trancher, le juriste, lui, est appelé à trancher, à résoudre un conflit. C'est à ce niveau qu'il est possible de situer la supériorité de la pensée juridique, laquelle « est liée à l'obligation qu'a le juge de trancher le litige et de le motiver » (p. 53). Quant à ses sources, la pensée juridique de Chaïm Perelman navigue entre Athènes, Rome et Jérusalem (pp. 53-68). Toutefois, au cœur de cette 
pensée, « se trouve la notion de logique juridique » (p. 54) dont Perelman a synthétisé le rôle dans « les ontologies juridiques, c'est-à-dire les types de systèmes juridiques distingués selon leurs sources du droit » (p. 54). Ainsi en relève-t-il les forces et les faiblesses. II s'agit d'Athènes, Rome et Jérusalem, autrement dit du droit de common law, du droit continental et du doit juif (ou talmudique).

Afin de pallier les inconvénients qui leur sont propres, chaque système juridique a mis en œuvre des techniques pour assurer l'acceptabilité de ses décisions. C'est cette acceptabilité de la décision de justice qui justifie l'apparition de ce que Chaïm Perelman appelle la « logique juridique » (p. 59) qu'il définit, d'après Stefan Goltzberg, « comme une sorte de menu dans lequel le juge sélectionne raisonnablement la technique qui conciliera le droit et l'acceptabilité de la solution » (p. 60).

Chaïm Perelman plaide pour un droit topique, lequel permet que des raisonnements apparemment contradictoires puissent se faire face sans se détruire. Ainsi peut-il remettre en question « la faisabilité et la légitimité d'un droit entièrement logique » (p.67), dès lors que tout est question de motivation de la décision. Cette motivation a, entre autres finalités, celle d'assurer la transparence de la décision judiciaire, de favoriser la prévisibilité du droit et de s'inscrire dans le projet d'une société démocratique (p. 70).

Un autre procédé, défini souvent en des termes vagues, mais central dans l'argumentation juridique, est la présomption. Pour Stefan Goltzberg, ce procédé jette « des ponts entre le raisonnement général et le raisonnement juridique » (p. 7l) ; et sa fonction « est de combler l'absence des faits et des vérités 》 (p. 72). Liées à l'expérience commune, les présomptions sont « des hypothèses, des suppositions, qui tablent sur ce qui est normal, qui permettent de mieux s'orienter mais qui n'en demeurent pas moins falsifiables » (p. 73). Elles peuvent donc être falsifiées par les faits. Dès lors, le droit distingue deux types de présomptions : les présomptions légales et les présomptions de l'homme. Chaïm Perelman porte plus son attention sur ces dernières « parce qu'elles relèvent du sens commun et qu'elles ne sont pas prévues comme telles par la loi » (p. 75). Plus fondamentalement, ces présomptions humaines sont « instituées pour assurer une valeur centrale : la sécurité juridique » (p. 76) et sont un élément de preuve pour celui à qui elles profitent. Toutefois, pour Stefan Goltzberg, sur cette question des présomptions, Chaïm Perelman « a plus posé le problème qu'il ne l'a clairement résolu » (p. 8I). Ce chapitre se termine par l'analyse de quelques arguments, spécialement les arguments a fortiori, a pari et a contrario, selon le classement de Chaïm Perelman. Les questions que ces arguments soulèvent sont : l'argument a fortiori relève-t-il de la logique ou de la rhétorique? Qu'est-ce qu'il suppose et quel statut serait-il possible de lui accorder? Enfin, quelle est en fait la force de ces arguments? S'agissant de cette force des arguments, Chaïm Perelman les explique respectivement par l'expertise, le précédent et les valeurs. Seslon Stefan Goltzberg, ces trois notions « jouissent de la même souplesse que la notion de raisonnable : aucune n'est définissable et encore moins prévisible. Dès lors, aucune de ces trois explications ne parvient à faire oublier les nombreux passages où Chaïm Perelman semble plaider pour un relativisme argumentatif » (p. 92). Tout compte fait, Stefan Goltzberg estime que Chaïm Perelman, dans sa tentative de faire un contrepoids au réductionnisme logique qui ne connaît que des arguments contraignants, a payé le prix d'un nivellement des arguments (p. 88).

De ce qui précède, il convient de remarquer que l'argumentation juridique de Chaïm Perelman a eu pour objectif « de fournir au juge les moyens de concilier le respect du droit et le respect des valeurs que le droit est censé défendre » (p. 95), en essayant d'éviter le plus possible deux pièges, celui du formalisme et celui de l'arbitraire (pp. 95-101). Ce qui conduirait le juge à des jugements raisonnables, justes. Bien que se trouvant au cœur de sa philosophie, la notion de raisonnable, n'est pas, d'après Stefan Goltzberg, « véritablement définie » (p. I0I). Elle dessine en revanche « les limites du champ du droit et du juste » (p. 102). Cette indéfinition du concept de « raisonnable » chez Chaïm Perelman conduit Stefan Goltzberg à parler d'une notion non opératoire, non définie (p. 104).

Malgré ces quelques réserves, on ne peut s'empêcher de parler d'un héritage perelmanien que l'auteur résume en quatre points (pp. 108-1 |4) : le pluralisme juridique et la conception large des sources, une approche topique de l'argumentation, le pragmatisme qui doit caractériser la science du droit ; et, enfin, la reconnaissance du droit comme lieu de conflit. Pour qui veut s'initier à l'argumentation juridique de Chaïm Perelman, voilà avec cet ouvrage un outil indispensable.

Julien Mumpwena Nsulung Université catholique du Congo, COG jmumpwena@gmail.com 